\section{(1) bsertbations}

ON THE

\section{NATURE OF CANCER.}

Br THE LATE JOHN MARSHALL, F.R.S., F.R.C.S., PRESIDENT OF THE GENERAL MEDICAL COUNCIL ; EMERITUS PROFESSOR OF SURGERY IN UNIVERSITY COLLEGE; CONSULTING SURGEON TO UNIVERSITY COLLEGE HOSPITAL.

THE late Mr. Marshall proposed to devote the following pages to the elucidation and amplification of certain statements and views which could only be but briefly expounded in an extempore lecture ${ }^{\perp}$ of one houx's duration. In the present posthumous lecture he has, furthermore, dealt briefly with several questions relating to cancer which were al together passed by in that lecture.

1. Definitions and nomenclature.-The confusion begotten of the threefold use of the term "cancer," sometimes to define a class, sometimes a genus, and sometimes a species of tumours, or even in two or three of these senses, or at all events in a general and a special sense in the same page, is much to be regretted; but it is perhaps hopeless to attempt to rid ourselves of it for the present. A trace of such confusion lurks in the title of this lecture and of its two predecessors ; for it is only by regarding the term "cancer" in one sense and the term "cancerous diseases" in another sense that the two terms become logically discreet, and subject to the copula "and." Otherwise the combination is mot merely tautological, but illogical ; for a "cancer" must be a "cancerous disease," and a "cancerous disease" must be "cancer." It might be well if, although as physicians and surgeons we are wholly unable to exclude the word "cancer" from our vocabulary, we could, under the guidance of the pathologist, more strictly limit its use. In practice, from kindly motives, the word "sarcoma" is frequently employed, when it is legitimate to do so, as a verbal mitigation of the more dreaded term. Doubtless, in ancient medicine and surgery, the earliest application of the term "cancer" was especially or chiefly to those forms of spreading and ulcerating growths which are typified by the hard, and hence socalled "scirrhus," tumours ; but it was also applicable, and was probably used, in regard to the softer and rarer forms of ulcerated new growths, later distinguished as encephaloid tumours ; and, furthermore, it was doubtless employed in relation to the worst forms of progressively eroding cutaneous ulcers. But the term "cancer" undoubtedly came also to be used in relation to the larger forms of those tumours which are now designated "sarcomas," especially to those which are prone to ulcerate or slough, or have actually undergone those morbid changes. This is abundantly manifest by the descriptions and illustrations contained in theses and systematic works dating from the period of the revival of learning down to the present day. Notwithstanding the precise differentiation now established on anatomical grounds between these several tumours, their general characteristics, pathological significance, and behaviour serve sufficiently to unite them under the one term of "invading or infecting tumours" But, it appears to me, a likeness or unlikeness in structure affords a more definite basis of a sound classification and just nomenclature than any resemblance or difference in their modes of life, propagation, or decay. The use of the term "true cancer," now sanctioned as a mode of distinction between the two chief and well-recognised divisions of these invading growths, is convenient, but unsatisfactory ; for, again, a "cancer" is a "true cancer," and a "true cancer" is a "cancer." Moreover, no one dreams of seriously employing the complementary opposite, "false cancer." For my own part, I cannot bring myself to designate any sarcoma which is derived from non-glandular tissue plements as a cancer. I prefer to adopt the subdivision of these "invading tumours" into two leading groups; $(A)$ the theliomas and $(B)$ the sarcomas, according as they are derived from the "covering or lining" constituents, or from the "substantial framework" constituents, of the body.

A. Theliomas, whatever may be their site or basis of nrigin in the body, are "cancers." They originate in or from parts of the body which are descended from one or other of the three so called embryonic layers of the blasto-

1 The Mortnn Lecture on Cancer and Cancerous Diseases, delivered at the Royal College of Surgeons of England on Nov. 18th, 1889 .

No. 35047 . derm-viz, the epi., the meso-, or the hypo blastic layers, and are either $(a)$ epitheliomas, (b) mesotheliomas, or $(c)$ hypotheliomas. 'The epitheliomas $(a)$ and the hypotheliomas (c) either atfect mere plain membranes or surfaces, thus producing flat masses-the so-called plaques; or they affect the elevations or projections of a cutaueous or mucous surface, and so give rise to warts, or papillomas; or they affect the minute involutions of those surfaces, such as simple or compound open follicles, branched or contorted tubules, or small racemose glands, whether these belong to the skin or to the mucous membranes ; or, lastly, they affect smaller or larger portions (never the whole) of the lobulated glands, such as the mammary gland, the salivary glands, the pancreas, the liver, the lungs, the testis, or the ovary, thus giving rise in thesesituations to more manifestand of ten very large tumours. In the case of the larger glands, and probably in smaller ones, the cancerous disposition may primarily affect the epithelium of the ducts or that of the acini of the gland, and thus either duct cancers or proper gland cancers arise. If the acini only are involved, an adenoid cancer is developed, which, indeed, constitutes a potentially, if not an actually invading adenoma. Lastly, the closed glands, such as the tonsillar and thyroid bodies and Peyer's patches, are liable to theliomatous disease. Mesotheliomas $(b)$, which as primary formations are rare, are represented by such theliomatous growths as can be distinctly shown to originate in sites acknowledged to be within the area of those parts of the body which are derived directly from the mesoblastic embryonic layer-such, for example, as any well-established cases of primary thelioma of the peri- and endo-cardium and the lining membrane of the bloodvessels, that of the lymphatic vessels and glands, and of the synovial membrane. These tumours must of course always be distinguished from deep-seated secondary growths, the products of invasion of an original epi- or hypo-thelioma. I believe there exists in every form of "thelioma," however minute, a substratum of modified basal tissue, not necessarily a distinct basal membrane which, as it seems to me, may probably be descended from the substance of the corresponding embryonic layer, whether epi-, hypo-, or meso-blastic; but the subject demands further investigation. I have long ago felt assured, from microseopic observations, that in every form of epithelioma and hypothelioma this modified basal tissue exists. It is obviously present in an elevated cancerous patch of skin or mucous membrane. It distorts a papilloma; it forms a loose and delicate stroma in soft gland, like the liver; and in an encephaloid growth it constitutes the firm and stubborn stroma of a scirrhous tumour ; and it may even form a chondroid or osteoid framework in an originally theliomatous growth. The variety of invading new growths, known as colloid cancers, whether derived from epiblastic, hypoblastic, or mesoblastic tissues, are colloid theliomas, having very special characters both as regards their peculiar cell elements and their interwoven stroma. In this respect they depart from the theliomas generally, in which, as a positive rule, the diseased and distorted individual cell elements maintain a general likeness to those of the healthy tissue in, npon, and from which they immediately spring.

$B$ The other group of invading tumours, the sarcomas, which originate in and from tissues which are especially derived from the mesoblastic embryonic layer, follow quite naturally the several varieties of those tissues. Snch, for example, are the sof 6 my xomas or mucoid tissue tumours, the round celled, spindle-celled, and fibroplastic growths or tibromas, the myomas or muscle tumours, the soft and hard chondromas, the lipomas, myelomas, and the open and compact osteomas. As is well known, two or more of these varieties of sarcomatous tissue frequently exist intermixed in the same grow th -an incident referable doubtless to their community of origin and type. Those interesting lower forms of tumour which consist of elements smaller and more single than of the proper tissues-such as tuberculous, gummatous, and inflammatory deposits-are necessarily excluded from special consideration here. Besides this, however, there are found occasionally mixed thelio-sarcomas-that is, tumours in which some portions have a theliomatous and other portions a sarcomatous structure, or in which these two forms of morbid tissue are in places closely and intimately blended. Seeing that the body itself is so composite an organism, and is descended from all three of the embryonic layers, the occurrence of these ( $A$ plus $B$ ) compound growths need not $f \mathrm{X}$ ite surpli-e. The primary separation of the germinatiog membrave of the ovam, whether into two, as ectoderm and endo- 
derm, or into three, as epiblast, mesoblast, and hypo. blast, is doubtless of the highest consequence and most essential character. A twofold division is, indeed, a mechanical necessity for any mucous membrane, and is naturally suitable for the foundation of a hollow organism; whilst a third, or mesial stratum, seems almost equally an unavoidable material consequence. But these necessary dispositions are not less wonderful on account of their simplicity; whilst such early, if not actually primal, arrangements would seem to have an obvious fundamental character and force, and to imply a kind of future controlling or hierarchical office and duty. From this point forward each lamina tends to breed true and keep true, and the distinction between them as to their constituents and functions respectively is henceforth maintained, and not easily, perhaps never actually, to be broken through. Within each layer the most complicated and yet definite, metamorphic, metabolic, and synthetic tendencies and processes are manifested and realised, but in each these are distinctly and regularly restricted. Definite evolution within them individually is the law for each; whilst the notion that a cross interference occurs, whether effected in a direct manner by simple metamorphosis of the tissue elements or by a process of previous reversion to a common and primitive uniformity of type and office, is difficult of acceptance, somewbat improbable, and at all events not proved; nor has it been actually shown that it is from hidden archaic protoblastic elements that the re. quisite varieties of forms are produced in late adult life. Certain elementary epithelial or hypothelial protoblasts may, indeed, be snpposed to shape themselves, if needful, into spherical, oval, squamous, polyhedral, columnar, or even ciliated forms; and, indeed, transitional forms of epi-and hypo-thelium are well known, and even occasional transmutations of form have been observed. A parallel instance of transmutation is to be seen in the flattening down and disappearance of cilia where the tracheal mucous membrane in the new-born kitten becomes subject to friction opposite the overlapping cartilages of the windpipe after respiration has been established. In the case of certain theliomas of primary origin, developed on the confines of the epiblastic and the bypoblastic regions of the body, the elementary constituents, though diseased and disfigured, still obey the particular type of the healthy tissue from which they have sprung, and, although conterminous, it must not here be concluded that the one kind of thelial element has been changed by contact, influence, or interactionary metamorphic power exercised upon it by the other. The bypothesis of the action of special antecedently existing germinal or seminal influence or force, giving rise to these transmutations, is needless to account for them, and in regard to any assumed transmutation of a mesoblastic elementary particle into a resemblance or copy of an epiblastic or hypoblastic element, the cure involvesastill moredifficultassumption. The further supposition that this effect is possible, either by a preliminary reversionary process, or by an undoing of the metamorphic process, is pure speculation. Direct observation is at fault. Mere deformation of neighbouring types of form, together with close contiguity or intermixture with lower ones, may account for appearances which might be attributed to subtle metamorphic change. The actual proof of positive transformation or interchangeableness is difficult to imagine, and is at present wanting. An approximation in form and character, as seen under the microscope, may obviously be delusive, and the evocation of a germinal or seminal influence of one tissue element over another, or even of traumatism upon one variety of neoplasm is merely speculative, and absolutely without foundation. Further facts and clearer demonstrations may some day be forthcoming. Meanwhile the limitation of the material involved, the definite nature of function, and the rigid exactitude of the evolutionary processes implied in the recently announced theory of Weissmann are altogether opposed to the idea that, when once the three layers of the protoblast are discriminated, any cross action or jumble of development from an epithelial cell to a connective tissue element is probable, or even possible, nor yet between a cancer cell and a round or spindle-shaped sarcoma element. Each kind of morbid growth continues true to itself, but intermixtures of them may and do occur in the highly composite animal frame.

From general considerations, as the foregoing, we may now turn to the examination of what are frequently termed more practical points. Nevertheless, no one part of this most important subject is without its close connexion with the rest, and those upon which I now wish to add a few short comments exhibit this inter-relationship in the highest degree.

As regards age, a sufficient regard, it seems to me, has not been paid to a possible source of general fallacy-namely, that facts are often derived from death records or mortality, without due reference to the duration of the disease. A further special fallacy is naturally the great difficulty of ascertaining the real date of the origin of the neoplasm: the fallacy still remains, even if the date of apparent commencement be, as is sometimes the case, taken as the starting point. Allowing for this, or even, I believe, without such strict allowance, my own experience would be, not from the fortieth to the sixtieth year, but from thirty-five to fifty, as the most frequent period of the onset of the malady.

As regards the influence of sex, with which that of special organisation is concerned, everyone will admit that women suffer more from true cancer than men; but that if sexual specialities be set aside men suffer more than women, as in the case of cancers of the lips, mouth, and alimentary canal generally. The most reliable numbers drawn from clinical experience are merely the result of so many asso. ciated and intervening provocative influences, and can only have an approximate relation to the truth. As to special organs, their derivation from one or other of the three primitive embryonic layers has the highest significance, for true cancer largely predominates in the epiblastic and the hypoblastic organs, as compared with the fully formed mesoblastic structures.

As to hereditary infuence, it is of undoubted and large significance. That, at least, is the personal experience would here put on record. The idea is old, and, indeed, is itself inherited; and it is faithfully enshrined in the well-known phrase, capable of a physical as well as a moral and social interpretation, "the ills which flesh is heir to." It is noteworthy to observe how its admitted effects are exaggerated and insisted on, or minimised and almost ignored, in view of the different opinions beld as to the causation of cancer itself. The constitutionalist inclines to exaggerate, the parasitist to minimise, the influence of inheritance. The absence of evidence of heredity may be due to want of knowledge or the concealment of knowledge on the part of a patient, or to the premature death from accident or other disease of individuals, who yet had a latent proclivity to cancer. On the other hand, the instances: on record of multiple inheritance in the members of the same family may be overvalued by some and undervalued by others; though they are certainly of real and grave significance. Hereditary influence most probably enters into the problem of the prevalence of cancer within certain localities or dis. tricts in which constant intermarriages probably occur generation after generation, and this may conduce to a special proclivity to the disease in certain self-limited races, as I have myself often observed amongst Jews; and on a larger scale in certain absolutely limited tribes or nations. The difficulties of following mentally an in. heritable materies morbi, in the shape of a substantive morbid molecule, is not greater than that of assuming the perpetuation of such a substantial modification of a tissue, which must be associated with a proclivity to a par. ticular disease. The student of the almost infinitely little of molecular constituency and of the almost infinite distance and number of unseen stars, and above all the mathematical mind, will find no inlerent difficulties in either of these conceptions. But until the cause of cancer itself is known, the nature and conditions of its inheritance cannot be defi. nitely indicated. At present my own impression, founded on experience, is that its influence is larger than that frequently or usually assigned to it. As to the personal equation due to original differences in the digestive, assimulative, and secretory functions or the actual metabolism of the body, it is quite incommensurable. So, too, the acquired peculiarities due to the antecedence or coexistence of a phthisical tendency, or of any stage of enthetic disease, cannot. yet be accurately estimated or even clearly distinguished. In the antagonism between a proneness to phthisis and a liability to cancer I am personally constrained to believe. Here the numerical exceptions seem to me to prove the rule. Again, an apparently increased personal liability may be really a diminished power of resistance, and vice versî́, and an apparently positive immunity may be quite accidental, and not due to any constitutional conditions. The alleged steady increase of cancer amongst ourown population 
at all periods of life, especially at the period of greater liability, and more amongst males than females, involves serious considerations. The disease is now said to prevail more in England than in Ireland; but, on the other hand, it has been pointed out that in the United States it is more prevalent amongst those of Irish and of German descent than amongst the rest of the population. Still, further researches on all these points are required.

The influences due to social status, and of the many conditions which accompany wealth or poverty, appear to me, in relation to the actual proportions of the rich and the needy, to be adverse to the well-to-do classes. Excess of diet, especially of animal food, used to be said to conduce to cancer; and carnivorous mammalia and birds, especially in a state of domestication, are known to suffer more than the herbivora. The latter, however, are killed earlier, not only for the necessities of the human race, but also for those of animals of prey. Tame and wild vegetable-feeders, as a rule, fall early victims to their predatory fellow creatures. Long-lived domesticated animals, even if herbivorous, suffer from some forms of cancerous disease; and I well remember dissecting and examining a wild hare, sent to my old master, Robert Liston, which had a well-marked soft adenoid tumour of the mammary glands on the left side of the body, which weighed more than two pounds. The genuine effects of habit and irritation were especially seen in regard to the former nse of the clay pipe, and are now shown in reference to the action of tobacco vapours on the tongue and palate. Many instances of both these facts are engraved upon my memory. But I would now add that $I$ have never met with a due inquiry into the effects of chewing tobacco. Is there any peculiarity in the mode of preparing tobacco for mastication and for smoking? Is there any mischievous empyreumatic evolution in the distilling action of the pipe or the cigar? Why did not or does not the use or the abuse of snuff give rise to the development of cancer in the noseof which I may say that I do not know of a single instance?

Finally, I have to note that the influence of occupation, and of its concomitant exposure to the effects of external agencies in relation to the provocation or prevention of cancer, has not been sufficiently investigated, more especially in regard to the continued inhalation or swallowing of vapours, or of minute particles of mineral substances contained in the air which workmen breathe, or the water they driok, or in the dust which lights upon their skin. Especially would I direct attention to the advisability of making an inquiry into the apparently bad or good effects of particular vapours or dust, which might contain zine, lead, copper, silver, mercury, antimony, or arsenic. This investigation should also extend to the influence of organic vapours or of dust, consisting of living or of dead organic matter.

Lastly, to revert to the real object of all such inquiryviz., the discovery of the causative agent in the pronuction of cancer from what appears to be healthy tissue, - I would arge upon our modern investigators the great desirability of comparing not merely the histological details of cancerous and normal structures, but to attempt the chemical analysis of the same. By a combination of all such methods of research we may ultimately remove the reproach of ignorance on this most common and most fatal disease.

\section{$=$}

\section{A CASE OF}

\section{HEPATIC ABSCESS IMPLICATING THE PLEURA, LUNG, KIDNEY, AND COLON; RECOVERY.}

By E W. ROUGHTON, B S , M D.LoND., F.R.C S , ASSISTANT SURGEOY TO THE ROYAL FREE HOSPITAL; SENIOR DEMOY STRATOR OF ANATOMY AND WARDEN OF THE COLLEGF AT ST. MARY'S HOSPIT.AL.

DURING the past few years the surgery of the thorax and abdomen has advanced with rapid strides, and perhaps of all the viscera contained within these cavities few of them have reaped more benefit therefrom than the liver. The following case will no doubt prove of interest to those who have worked in this field.

The patient, who was a strong athletic gentleman aged twenty-three vears, was taken ill with febrile symptoms on Jan. lith, 1889, at Kandy, in Ceylon. In a few days pain over the liver and a bulging under the costal margin indi- cated the presence of an hepatic abscess. On Feb. lst the abscess was opened and ten or twelve ounces of pus evacuated. The drainage does not seem to have been efficient, inasmuch as the temperature rose to between $101^{\circ}$ and $102^{\circ}$ in the evening. However, by March lst the abscess had healed np, but still the temperature kept above normal. This led to an examination of the chest, resulting in physical signs of pleurisy with effusion being cletected on the right side. Up to this time there had been no cough, dyspnoea, or other chest symptom. On March 16 th twenty-seven ounces of clear serum were drawn from the right side of the chest by an aspirator inserted near the angle of the seapula. On March 26th the same operation was repeated, and with similar result. It appears that the physical sions of effusion continued, as he was aspirated again on April 4th, 8th, and $12 \mathrm{~h}$, close to the original place, but on these occasions nothing was withdrawn. These aspirations had been performed on board ship during his journey to England, where he arrived on April 13th. On the next day he came under my care, and I then made the following note: "Strongly built man, but decidedly emaciated; appetite good. Scar over liver in nipple line, just under costal margin. Liver dulness extends one inch below costal margin. On the rigat side of the cuest there is dulness below the nipple and behind the anterior fold of the axilla; posteriorly, the dulness extends as high as the spine of the scapula. Over the dull area there is almost complete absence of respiratory murmur, vocal resonance, and vocal vibration. Heart not displaced; left lung normal ; slight hacking cough, with greenish expectoration." The opinion that I formed at that time was that the physical signs indicated a pleurisy with effusion due to extension of inflammation from the liver through the diaphragm. As be had been greatly shaken during his journey to London, I thought it wisest to adopt no active treatment at once, but to allow him a few days rest in bed. On the evening of April 18th the temperature rose to $101.2^{\circ}$, and was followed by profuse perspiration. The perspirations were repeated nightly, and on the evening of the 21 st the temperature reached $102 \cdot 2^{\circ}$. The following day it was considered advisable to explore the chest with a needle, and (after consultation with Dr. Gee) pus was tapped in the right axilla, a puncture near the angle of the scapula having been made without result. Under chloroform an incision was made near the posterior fold of the axilla, and an inch of the sixth rib excised subperiosteally. About twenty-five ounces of pus were evacuated. The finger inserted into the opening discovered two cavities, one extending forwards towards the nipple, and the other downwards towards the costal margin, partially separated from one another by $n$ septum. As the incision seemed to have struck the most dependent part of the cavity, no counter-opening was made. A large drainage-tube was inserted. The immediate result of the operation was very satisfactory, the temperature falling to normal and remaining so until April 28th, when, without discoverable cause, it ran up to $101 \cdot 2^{\circ}$. Thinking there might be some retention or decomposition of discharge, I explored the cavity under chloroform and washed it out with a weak solution of carbolic acid on the $29 \mathrm{th}$. This was not attended by any gocd result, as next day the temperature had reached $103^{\circ}$. The fever, however, gradually subsided without other treatment and without obvions reason, so that on May 7th the temperature was normal, and remained so for more than a week. On May 16th, whilst the cavity was being washed out, he suddenly coughed very violently and said he tasted the carbolic acid which was being used. On the evening of the 18 th the temperature rose to $101 \cdot 2^{\circ}$ and increased every evening, till on the 21 st it was $103^{2}$. On the 25 th he had a severe attack of coughing, and said he had a "rotten taste" in his mouth. When he coughed a distinctly fetid odour could be detected, although his expectoration was almost free from smell. The discharge from the wound remained quite sweet. Judging that some portion of the cavity bad become shut off from the external opening and had established a communication with the lung, and so become fetid, I had the patient put under the influence of chloroform again and inserted my finger into the external opening. The cavity seemed to have contracted to little more than the track oscupied by the drainage-tube, but at the end of the track a small hole abont large enough to admit a good-sized probe was found. When this was enlarged with dressing forceps, it was found to lead into a cavity about an inch in diameter. presumably partly in the lung and partly in thickened 